\title{
Cat-state generation and stabilization for a nuclear spin through electric quadrupole interaction
}

\author{
Ceyhun Bulutay* \\ Department of Physics, Bilkent University, Ankara 06800, Turkey \\ (Received 20 October 2016; revised manuscript received 19 January 2017; published 11 July 2017)
}

\begin{abstract}
Spin cat states are superpositions of two or more coherent spin states (CSSs) that are distinctly separated over the Bloch sphere. Additionally, the nuclei with angular momenta greater than $1 / 2$ possess a quadrupolar charge distribution. At the intersection of these two phenomena, we devise a simple scheme for generating various types of nuclear-spin cat states. The native biaxial electric quadrupole interaction that is readily available in strained solid-state systems plays a key role here. However, the fact that built-in strain cannot be switched off poses a challenge for the stabilization of target cat states once they are prepared. We remedy this by abruptly diverting via a single rotation pulse the state evolution to the neighborhood of the fixed points of the underlying classical Hamiltonian flow. Optimal process parameters are obtained as a function of electric field gradient biaxiality and nuclear-spin angular momentum. The overall procedure is seen to be robust under 5\% deviations from optimal values. We show that higher-level cat states with four superposed CSS can also be formed using three rotation pulses. Finally, for open systems subject to decoherence we extract the scaling of cat-state fidelity damping with respect to the spin quantum number. This reveals rates greater than the dephasing of individual CSSs. Yet, our results affirm that these cat states can preserve their fidelities for practically useful durations under the currently attainable decoherence levels.
\end{abstract}

DOI: 10.1103/PhysRevA.96.012312

\section{INTRODUCTION}

In the midst of the so-called second quantum revolution [1], nuclear-spin systems have been among the first to be proposed and tested $[2,3]$. In due course, the overwhelming majority of implementations have utilized an ensemble of nuclear spins which stems from the established bulk nuclear magnetic resonance-based manipulation and detection schemes [4]. For quantum information processing, working with a single spin is desirable to alleviate issues arising from ensemble averaging, however, it was initially hindered by the poor detectability [5]. More than two decades ago the single electron spin detection within a host crystal was achieved [6,7]. In the case of a single nuclear spin the remaining challenge was its about two thousand times smaller magnetic moment compared to electron [4]. The breakthrough came with the aid of optically detected electron nuclear double resonance [8]. Subsequently, the optical readout of a single nuclear spin in a nitrogen-vacancy (NV) defect center in diamond was announced $[9,10]$. The next milestone reached on this front was the single-shot readout of a single nuclear spin, again within the NV system at room temperature [11-13]. As other solid-state examples, and using an electrical readout scheme, the implementations on a $\mathrm{Tb}$ nuclear spin of a single-molecule magnet [14] and a ${ }^{31} \mathrm{P}$ donor nuclear spin in silicon [15] can be mentioned; for a very recent review, see Ref. [16].

Such a control on the single-spin level in a solid-state system opens enormous opportunities for quantum technologies [1]. Mainly because spin offers an excellent framework for demonstrating interesting quantum states and effects such as coherent states [17], squeezing [18,19], to name but a few. An important class in quantum mechanics is the cat state which corresponds to macroscopically separated

*bulutay@fen.bilkent.edu.tr coherent superpositions of coherent states [20,21]. Therefore, its realization in various spin systems has been the aspiration for a number of proposals lately, such as the generation of spin cat states in Rydberg atoms [22], in Bose-Einstein condensates [23], in a finite Kerr medium for the big spin-qubit [24] or spin-star model [25]. In terms of applications, spin cat states are suggested for high-precision measurements via dissipative quantum systems of Bose atoms [26]; an exhaustive review of quantum metrology is available from Ref. [27]. Unfortunately, these are either of model level [24,25], or based on atomic nonlinearities [27], arising from Rydberg blockade [22], or collisional effects in Bose condensed atoms $[23,26]$. Therefore, for the case of a nuclear spin in a solid-state environment these recipes are of no avail.

In this work our aim is to present a simple means to generate different kinds of cat states on a single nuclear spin by harnessing the quadrupole interaction (QI) [28,29] which intrinsically operates on the quadrupolar nuclei, that is, with spin quantum number greater than $1 / 2$. Motivated by a recent experimental exposition of squeezing with spin-7/2 nuclei [30], this work builds upon our prior study where we have shown that the generic QI supports continuous tuning of squeezing from one-axis to two-axis countertwisting limits [31]. We confine ourselves to the spin values between 1 and $9 / 2$ that correspond to the range of abundant isotopes of quadrupolar nuclei. We adopt new concepts developed for freezing the spin squeezing [32-34], in our case to stabilize the cat states once they are produced. The robustness of our scheme is checked under various drifts or errors in the process parameters [35]. Furthermore, going up to the next level in hierarchy, we consider the production of the superpositions of spin cat states that is essential for the quantum error correction against spin flips without revealing the registered quantum information [36]. The kind that we discuss corresponds to a rotating spin cat state superposed to a fixed counterpart, enabling a relative phase accumulation, that is composed 
of the so-called moving coherent states [37]. We assure the longevity of these states by exhibiting their resilience to phase decoherence under realistic conditions.

The paper is organized as follows. In Sec. II we present the background theoretical information as well as our notation on QI, coherent spin and cat states, measures used for assessment, decoherence model, and the phase portraits of the QI Hamiltonian. In Sec. III we report our results starting with the overall operation, followed by optimization of performance, and its sensitivity analysis; we then discuss the extension of cat-state generation to their superpositions and how decoherence affects the cat states. We also comment on practical aspects and potential applications. Our main findings are summarized and suggestions for future directions are outlined in Sec. IV. The Appendix section contains the derivation of the closed-form expression for the time evolution operator under a certain case that is required in the main text.

\section{THEORY}

\section{A. Quadrupole spin Hamiltonian}

Nuclei with spin $I \geqslant 1$ are named as quadrupolar because of their multipolar charge distributions. This makes them susceptible to electric field gradients (EFG) [28,29]. The latter is routinely present within a solid-state matrix, predominantly being caused by strain [38]. The EFG at a nuclear-spin site is described by a second-rank tensor involving second-order spatial derivatives of the crystal potential $V$, $V_{i j} \equiv \partial^{2} V / \partial x_{i} \partial x_{j}$, which becomes diagonal for a particular orientation of coordinate axes. This is referred to as the principal EFG axes, which is what we shall use throughout this work. Here, as a convention, the Cartesian axes are labeled in the way that the nonvanishing EFG components obey the ordering $\left|V_{z z}\right| \geqslant\left|V_{y y}\right| \geqslant\left|V_{x x}\right|$.

The EFG acts on the spin degrees of freedom of a quadrupolar nucleus as governed by the Hamiltonian [28]

$$
\hat{H}_{\eta}=\frac{h f_{Q}}{6}\left[3 \hat{I}_{z}^{2}-\hat{I}^{2}+\eta\left(\hat{I}_{x}^{2}-\hat{I}_{y}^{2}\right)\right]
$$

where $h$ is Planck's constant and $f_{Q}$ is the quadrupole linear frequency controlled by the EFG major principal value, $V_{z z}$. Since we shall not consider any other steady term in the Hamiltonian, $f_{Q}$ will serve for setting the time scale of the dynamics; typical values will be stated when we discuss decoherence processes. The magnitude of spin angular momentum vector $I$ is conserved, and as in our prior work [31], this term can be dropped from dynamics at will. Note that we parametrize the Hamiltonian with respect to $\eta=\left(V_{x x}-V_{y y}\right) / V_{z z}$, which defines the degree of biaxiality of the EFG [28,29], and as we shall see, plays a central role for the cat-state generation and stabilization. It is confined to the range $[0,1]$ : the lower limit corresponds to a cylindrically symmetric EFG distribution, while the upper limit $\eta=1$ can be realized, for instance, in two-dimensional materials [31].

\section{B. Coherent spin and cat states}

A coherent spin state (CSS) centered around the spherical angles $(\theta, \phi)$ can be obtained from the $z$-oriented Dicke spin state $\left|I, I_{z}=I\right\rangle$ via

$$
|\theta, \phi\rangle=\exp \left[i \theta\left(\sin \phi \hat{I}_{x}-\cos \phi \hat{I}_{y}\right)\right]|I, I\rangle,
$$

where the operator on the right performs rotation around the axial vector $(\sin \phi,-\cos \phi, 0)$ by an angle $\theta$ [39]. For convenience we shall denote the CSS located around the six axial Cartesian directions over the Bloch sphere as $| \pm X\rangle$, $| \pm Y\rangle$, and $| \pm Z\rangle$. We emphasize that the Cartesian directions here are not arbitrary but are based on EFG principal axes. Once again, in relation to the Dicke state, $|j, m\rangle$ as labeled with total angular momentum $j$ and its quantization-axis projection $m$, we have for the $z$ quantization axis $| \pm Z\rangle=|I, \pm I\rangle$, while $| \pm X\rangle$ and $| \pm Y\rangle$ are their rotated forms around the $y$ and $x$ axes, respectively.

For quantum metrology and other quantum information technologies, it is the coherent superpositions of such CSSs that are of importance, especially if they correspond to macroscopically distinguishable superpositions, so-called cat states [40]. Historically, the even and odd cat states were first to be introduced, having the forms $\mathcal{N}[|\alpha\rangle+|-\alpha\rangle]$ and $\mathcal{N}[|\alpha\rangle-|-\alpha\rangle]$, respectively [20], where $|\alpha\rangle$ denotes a generic coherent state [41]. This is followed by the socalled Yurke-Stoler state, $\mathcal{N}[|\alpha\rangle \pm i|-\alpha\rangle]$ [21]. In these expressions, $\mathcal{N}$ is the normalization factor, which is different for each case; in the remainder of this work, for brevity we drop them from our subsequent notations. Additionally, in our following discussion, we prefer the terms equator-bound and polar-bound target cat states for $\left[|Y\rangle+e^{i \varphi}|-Y\rangle\right]$ and $\left[|Z\rangle+e^{i \varphi}|-Z\rangle\right]$, respectively, according to where the cat pair is located on the Bloch sphere. The remaining $x$ axis, paired with the minor EFG component, will serve as the main rotation axis. Such coherent superpositions of maximally separated two CSS over the Bloch sphere will be termed as $N=2$ cat states. In the Results section we also introduce the coherent superpositions of two maximally separated $N=2$ cat states making up a $N=4$ cat state.

\section{Measures}

To quantify how closely a generated state $|\psi\rangle$ reproduces a certain target state $|\beta\rangle$, a common measure is the fidelity which for such pure states becomes simply $F=|\langle\beta \mid \psi\rangle|$ [42]. Alternatively, rather than comparing with a fixed target state, one can use absolute macroscopicity measures [43]. These are generally based on the quantum Fisher information in regard to an operator/measurement $\hat{A}$, which reduces for a pure state $|\psi\rangle$ (as invariably considered in this work) to $\mathcal{F}(\psi, \hat{A})=4 \mathcal{V}_{\psi}(\hat{A})$, where $\mathcal{V}_{\psi}(\hat{A})=\left\langle\psi\left|\hat{A}^{2}\right| \psi\right\rangle-\langle\psi|\hat{A}| \psi\rangle^{2}$ is the variance. For a spin- $I$ system the effective size is then defined as

$$
N_{\mathrm{eff}}^{\mathrm{F}}(\psi)=\max _{\hat{A} \in \mathcal{A}} \mathcal{F}(\psi, \hat{A}) /(2 I),
$$

where one maximizes over operators within the relevant set $\mathcal{A}$. To quantify the degree of catness of a superposed state $\left|\psi_{S}\right\rangle=$ $\left(\left|\psi_{a}\right\rangle+\left|\psi_{b}\right\rangle\right) / \sqrt{2}$, the relative quantum Fisher information (rQFI) has been proposed [44] as

$$
N_{\mathrm{eff}}^{\mathrm{rF}}\left(\psi_{S}\right)=\frac{N_{\mathrm{eff}}^{\mathrm{F}}\left(\psi_{S}\right)}{\left[N_{\mathrm{eff}}^{\mathrm{F}}\left(\psi_{a}\right)+N_{\mathrm{eff}}^{\mathrm{F}}\left(\psi_{b}\right)\right] / 2} .
$$


For pure states, and choosing as the relevant interferometric measurement operators the spin along direction $u$ (i.e., $\hat{I}_{u}$ ), each maximization in Eq. (3) becomes trivial, yielding

$$
N_{\mathrm{eff}}^{\mathrm{rF}}\left(\psi_{S}\right)=\frac{2 \mathcal{V}_{S}\left(\hat{I}_{S}\right)}{\left[\mathcal{V}_{a}\left(\hat{I}_{a}\right)+\mathcal{V}_{b}\left(\hat{I}_{b}\right)\right]}
$$

The variance for a CSS is simply $I / 2$, and in the case of a diametrically opposite cat state, for instance, choosing one of the target states mentioned above, $\left|\psi_{S}\right\rangle \rightarrow\left[|Z\rangle+e^{i \varphi}|-Z\rangle\right]$, we have $\hat{I}_{S} \rightarrow \hat{I}_{z}$, which yields $\mathcal{V}_{S}\left(\hat{I}_{S}\right)=I^{2}$; therefore, the maximum value of $N_{\mathrm{eff}}^{\mathrm{rF}}$ becomes $2 I$, which indeed corresponds to largest possible separation over the Bloch sphere, namely, its diameter. Thus, to quantify the catness of an evolving state $\psi$ for a spin measurement along a direction $u$, we use normalized rQFI as

$$
\bar{N}_{\mathrm{eff}}^{\mathrm{rF}}(\psi)=\frac{\mathcal{V}_{\psi}\left(\hat{I}_{u}\right)}{I^{2}},
$$

which ranges between 0 and 1 .

\section{Accounting for decoherence}

There is a well-defined phase relation among the constituent CSSs reflecting the coherence of the superposition. As such, they are particularly vulnerable to phase noise. The resultant decoherence can be tracked via the system density operator using the Lindblad master equation [42]

$$
\begin{aligned}
\frac{d}{d t} \hat{\rho}_{S}(t)= & -\frac{i}{\hbar}\left[\hat{H}, \hat{\rho}_{S}(t)\right] \\
& +\sum_{m=1}^{2 I}\left[\hat{L}_{m} \hat{\rho}_{S}(t) \hat{L}_{m}^{\dagger}-\frac{1}{2}\left\{\hat{L}_{m}^{\dagger} \hat{L}_{m}, \hat{\rho}_{S}(t)\right\}\right],
\end{aligned}
$$

where $\hat{\rho}_{S}$ is the nuclear-spin density operator, and [,] and $\{$, represent commutator and anticommutator, respectively. $\hat{L}_{m}$ is a so-called Lindblad operator characterizing the nuclear spin's coupling to its environment [42]. For the phase-flip channel of a spin- $I$ system they can be extracted from the associated Kraus operators [45] as

$$
\hat{L}_{m}=\sqrt{\frac{(2 I) !}{m !(2 I-m) !}\left(\frac{1-e^{-\gamma}}{2}\right)^{m}\left(\frac{1+e^{-\gamma}}{2}\right)^{2 I-m}} \hat{I}_{z}^{m},
$$

where $\gamma=1 / T_{2}$ is the dephasing rate, with the well-known coherence dephasing time constant being $T_{2}$, which is routinely measured with spin-echo techniques [4]. Even though we shall be using this full Lindblad set, it can be readily verified that in the weak damping limit the Lindblad operators reduce to a single one $\sqrt{\gamma I} \hat{I}_{z}$, as considered in Ref. [31].

\section{E. Fixed points and their biaxiality dependence}

In the case of spin squeezing, the maximally squeezed quadrature is attained only for an instant over each (quasi-) period of the cycle [31]. To break away from this regime with large swings, Kajtoch et al. proposed to apply a rotation operation when maximum squeezing is reached and transfer the subsequent flow to regions around the fixed points of the classical Hamiltonian, where oscillation amplitudes can be
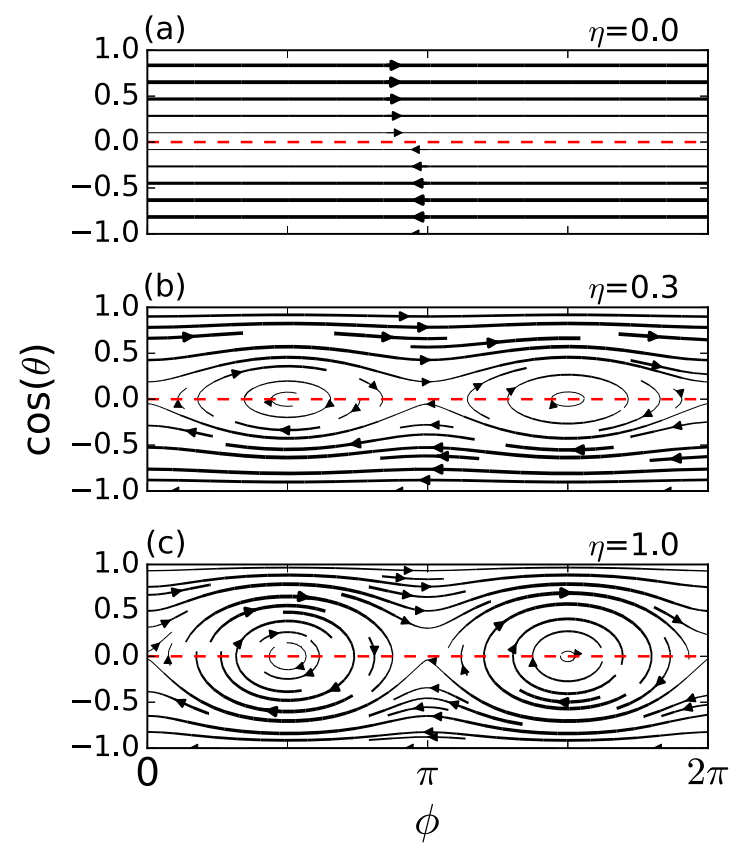

FIG. 1. Phase portraits obtained from Eqs. (10) and (11) for three different $\eta$ values. Dashed red lines mark the equator on the Bloch sphere. The thickness of the lines is proportional to the speed of the flows.

highly suppressed [34]. To implement this recipe for the QI under an arbitrary biaxiality $\eta$, we need the associated fixed points [46].

For a classical spin vector pointing toward the $(\theta, \phi)$ direction, the QI Hamiltonian in Eq. (1) takes the form

$$
H_{\eta}(\theta, \phi)=\frac{h f_{Q} I}{6}\left[3 \cos ^{2} \theta+\eta \sin ^{2} \theta \cos 2 \phi\right],
$$

through which the Hamilton equations of motion are obtained for the canonically conjugate variables $\left(\phi, P_{\phi} \equiv \cos \theta\right)$ as

$$
\begin{gathered}
\dot{\phi}=\frac{h f_{Q} I}{3} P_{\phi}(3-\eta \cos 2 \phi), \\
\dot{P}_{\phi}=\frac{h f_{Q} I}{3} \eta\left(1-P_{\phi}^{2}\right) \sin 2 \phi .
\end{gathered}
$$

The corresponding phase portraits are shown in Fig. 1 for three different $\eta$ values. Two stable center fixed points lie at the poles $(\theta=0, \pi)$ for any $\eta$. Additionally, for the case of $\eta=0$ the whole equator line $(\theta=\pi / 2)$ turns into fixed "points", whereas for $\eta \neq 0$ they reduce solely to four points at the $\pm x$ and $\pm y$ axes i.e., $\phi=\{0, \pi, \pi / 2,3 \pi / 2\}$. In the latter case, $\phi=\{0, \pi\}$ are unstable, and those at $\phi=\{\pi / 2,3 \pi / 2\}$ are of stable-center-type fixed points. In other words, for non-zero $\eta$ the stable fixed points over the Bloch sphere are positioned at $\pm y$ and $\pm z$ axes that we term as equator and polar bound, respectively.

\section{RESULTS}

\section{A. Basic operation}

To demonstrate the overall procedure, we consider a $5 / 2$ spin with an initial CSS lying on the $+x$ axis of the Bloch sphere, i.e., $|+X\rangle \equiv\left|\theta_{C S S}=\pi / 2, \phi_{C S S}=0\right\rangle \quad[$ Fig. 2(a)]. 


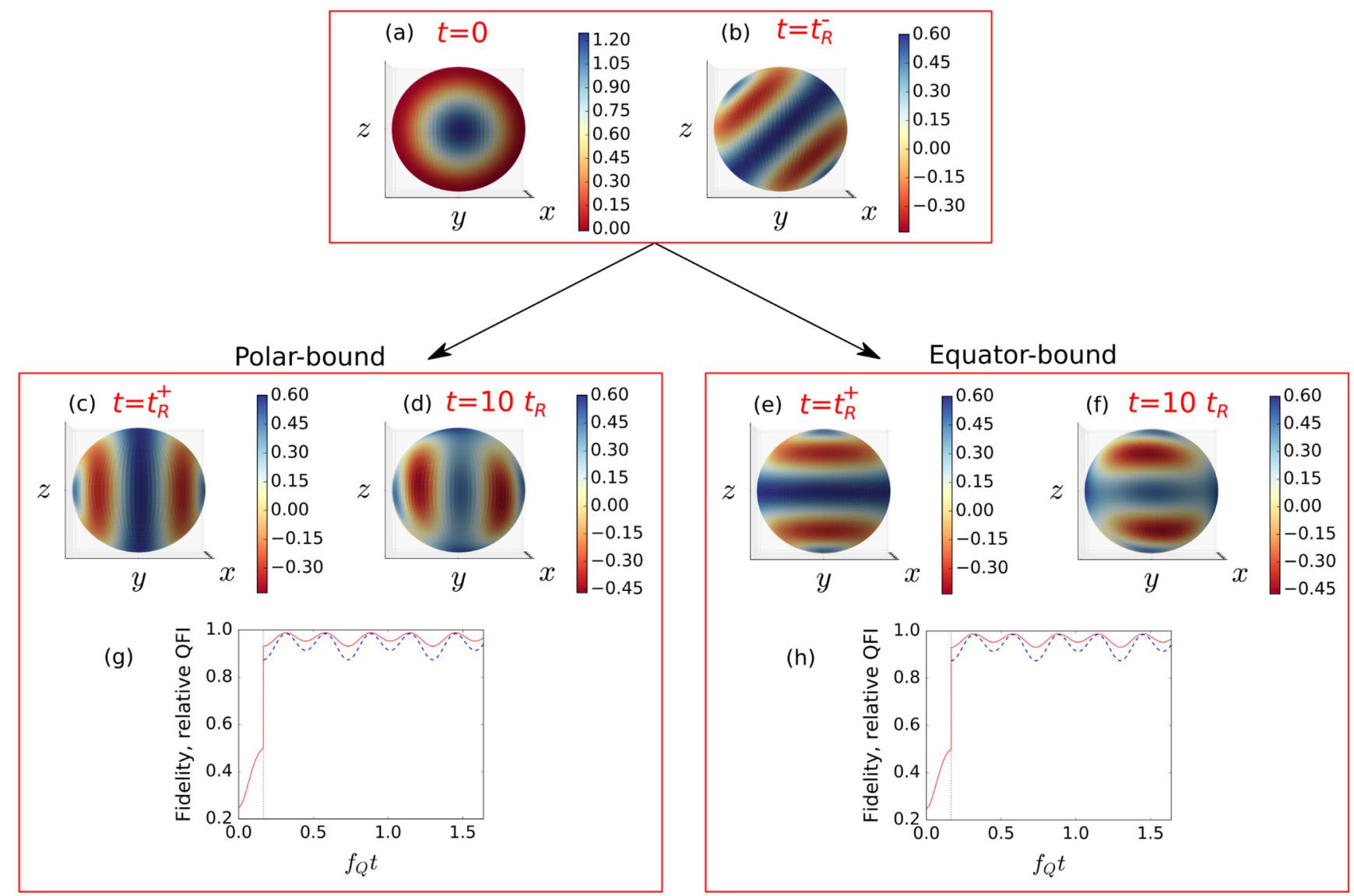

FIG. 2. The spin Wigner quasiprobability distributions at the main stages of the procedure starting from (a) an initial CSS, (b) just before the rotation instant $t=t_{R}^{-}$, (c)+(e) just after rotation to pole and equator planes $t=t_{R}^{+}$, (d)+(f) much later at $t=10 t_{R}$. Items (g) and (h) show the fidelity (solid red line) and normalized rQFI [see, Eq. (6)] (blue dashed line) for the polar- and equator-bound evolutions. A 5/2-spin with $\eta=1$ is considered. Vertical dotted lines mark the instances of the rotation pulses.

Under the action of $\hat{H}_{\eta}$ (for this example, $\eta=1$ ), it first goes through a squeezing stage, with the antisqueezed axis having rotated by about $\pi / 4$ from the equatorial plane over the Bloch sphere [Fig. 2(b)]. We terminate this regime suddenly by applying a rotation around the $+x$ axis that aligns the spin distribution elongation toward either the polar [Fig. 2(c)] or equatorial plane [Fig. 2(e)], coinciding with the two fixed points of the QI Hamiltonian, as discussed in the previous section. Hence, further evolution of the dynamics gets localized around the two antipodal fixed points, giving rise to either polar-bound $[| \pm Z\rangle$, see Fig. 2(d)] or equator-bound $[| \pm Y\rangle$, see Fig. 2(e)] cat states. For all cases, we resort to spin Wigner quasiprobability distribution plots $[47,48]$, which is sensitive to phases [41]. Observe that in between these antipodal regions, additional fringes exist, the hallmark of quantum coherence [49], as colloquially referred to as the smile of the cat [50].

The degree of success is quantified with the fidelity [Figs. 2(g) and 2(h)], typically reaching a maximum value of around 0.95 and a ripple of about 0.05; see Eqs. (12) and (13) below. The normalized rQFI calculated by Eq. (6) (shown by dashed lines) follows the same behavior of the fidelity but with a larger ripple. As the rQFI measure simply tracks the separation of the constituent cat states and is not anchored to a target (unlike fidelity), the valuable conclusion this provides is that concerted deviation from unity under both measures cannot originate from simply a rigid oscillation around the target state.

\section{B. Search for optimality}

For each quadrupolar spin from $I=1$ to $9 / 2$, we optimize with respect to the time instant $t_{R}$ and the angle of the rotation $\theta_{R}$ that will orient the major axis of the spin distribution toward the fixed points on either the poles ( $\pm z$ axes) or equator ( $\pm y$ axes). We also let the phase angle $\varphi$ between the constituent CSSs of the target cat states $\left[|Z\rangle+e^{i \varphi}|-Z\rangle\right]$ and $\left[|Y\rangle+e^{i \varphi}|-Y\rangle\right]$ to be yet another optimization parameter. Our two optimality criteria are

$$
\begin{gathered}
F_{\max }=\max F, \\
F_{\text {ripple }}=(\max F-\min F) / 2,
\end{gathered}
$$

that is, high fidelity with the associated target cat states and low ripple around the mean fidelity once in the stabilization stage, i.e., after the rotation instant. We assign the relative weights 0.55 and 0.45 to these two goals, respectively, and obtain corresponding optimal cat-state generation and stability performances. 
(a) Polar-bound
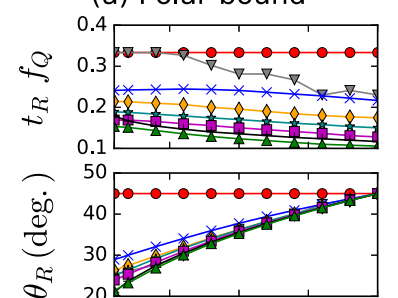

$1.0+0.0+2$
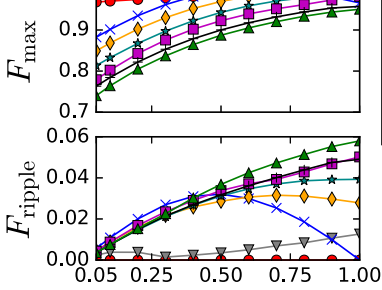

$\eta$ (b) Equator-bound

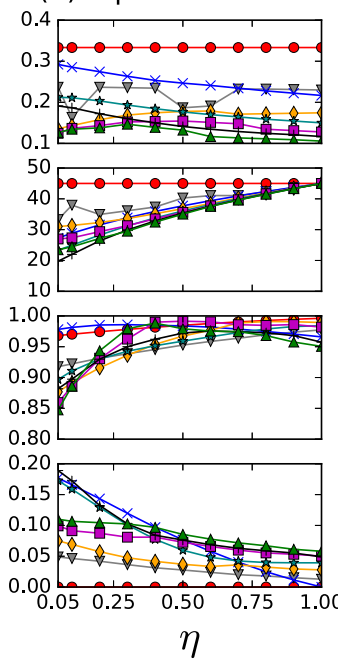

FIG. 3. Rotation instants and angles (in degrees) for the optimal cat-state generation and stabilization as quantified by maximum fidelity $F_{\max }$ [Eq. (12)] and its ripple $F_{\text {ripple }}$ [Eq. (13)] for polarand equator-bound cases.

For the polar-bound targets, this procedure culminates with strictly even cat states, whereas the equator-bound ones exhibit a spin- $I$ dependent phase angle, $\varphi=\pi I$, that is, for integer spin nuclei the cat states produced are of the same parity with $I$, and for all half-integer spins Yurke-Stoler-type cat states are generated. Figure 3 displays these results as a function of the QI biaxiality parameter $\eta$, the variation of maximum fidelity [Eq. (12)], its ripple [Eq. (13)], the instants of optimal rotation pulse, and the angles around the $+x$ axis required to orient them to the appropriate target planes. For both polar- and equator-bound cases, fidelity drastically drops when the uniaxiality of QI increases, i.e., $\eta \rightarrow 0$; however, for the former the ripple in the fidelity also decreases. This correlates with the fact that the equatorial fixed points soften as $\eta \rightarrow 0$. In the opposite limit of $\eta \rightarrow 1$, which corresponds to two-axis countertwisting [31], the optimal rotation angle goes to $\pi / 4$ for all cases, in accordance with the findings of Kajtoch et al. [34]. In general, as the spin- $I$ value increases the maximum fidelity reduces. In this regard, the $I=1$ case appears to show the best performance. However, $I=1$ is actually an outlier with respect to the higher spins, showing no dependence to $\eta$ at all. This three-level system also has a similar peculiarity in spin squeezing with exact vanishing of uncertainty in one of the quadratures [31]. It remains to be seen whether these seemingly impressive $I=1$ performances can be of any practical relevance.

\section{Sensitivity}

If the attained optimal conditions in Fig. 3 are only achievable for a very narrow range of parameters, it will hamper the practical utility of the proposed cat-state generation and storage. Therefore, in this section we present the sensitivity analysis around the operating points. For this purpose, we choose $I=3 / 2$ and $\eta=0.3$, where both a very high fidelity and low ripple values were observed, especially for the polar-bound case (Fig. 3). The instant when the rotation pulse

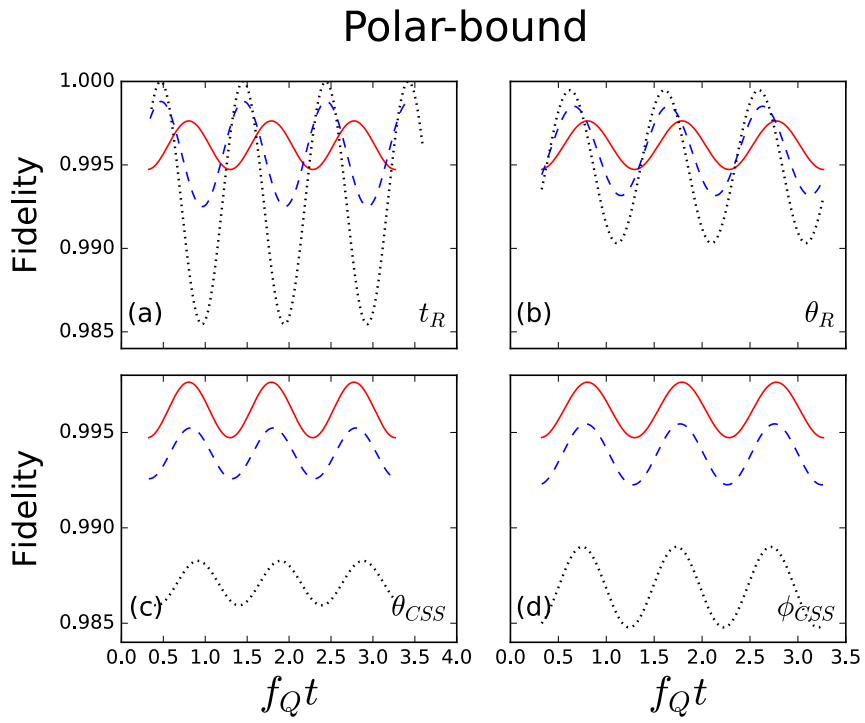

Equator-bound
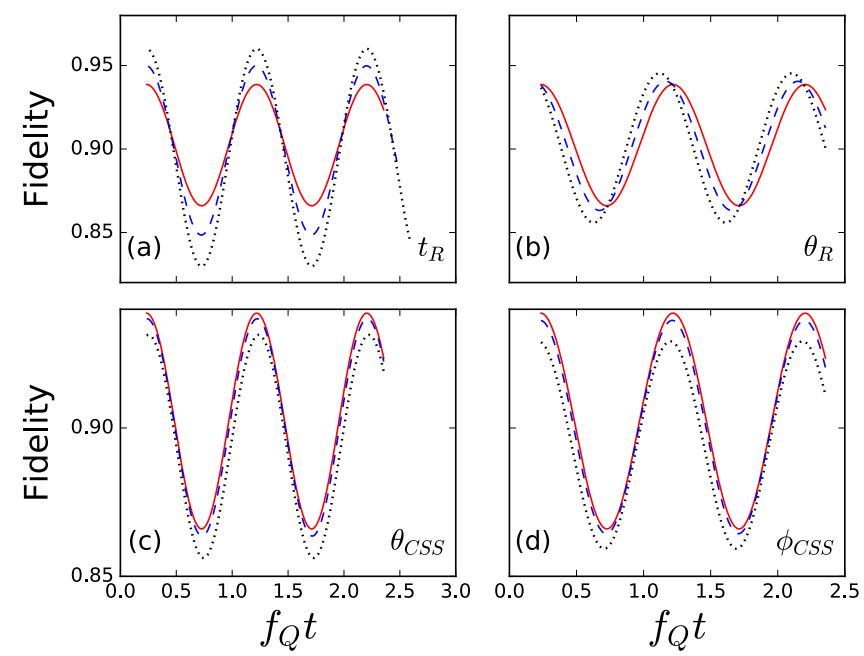

FIG. 4. For polar- and equator-bound cases, sensitivity of optimal fidelities (red solid line) under 5\% (blue dashed line) and 10\% (black dotted line) deviation in the parameters of (a) rotation instant, (b) rotation angle, (c) polar, and (d) azimuthal offsets in the initial CSS. $I=3 / 2$, and $\eta=0.3$ is considered.

is applied, $t_{R}$ and the amount of rotation angle $\theta_{R}$ are the two main parameters here. Additionally, we consider unintentional displacements from the assumed location of the initial CSS, $|X\rangle$ along the polar $\theta_{C S S}$ and the azimuthal $\phi_{C S S}$ angles, as would be caused when the quadrupolar principal axes are not properly aligned with the CSS or rotation axes. This can be termed as the preparation error, encountered, for instance, in the NV centers [12]. In Fig. 4 we compare the time-dependent fidelity of the optimal case with those under 5\% and $10 \%$ deviations in each of these parameters. In general terms, a change in $t_{R}$ predominantly increases the ripple around the same mean fidelity value, and this parameter shows higher sensitivity than $\theta_{R}$ in the same range. $\theta_{C S S}$ and $\phi_{C S S}$ offsets usually result in the overall decrease in the fidelity without a significant change in the ripple. In any case, it is assuring that 
the overall performance does not bare a drastic dependence on the chosen operating parameters. Especially, a 5\% mismatch from optimal parameters leads to tolerable implications.

\section{D. $N=4$ cat-state generation}

Now, we would like to investigate the generation of the so-called $N=4$ cat state $[36,37,51]$ by superposing equatorand polar-bound cat states. For this purpose, we can start with either of these cat states (production of which requires one pulse) and through a second pulse rotate it by $\pi / 2$ back on to the $x$ axis, reproducing the $N=2$ cat state $[|X\rangle+|-X\rangle]$ with a high fidelity. Then, under $\hat{H}_{\eta}$, the time evolution of these antipodal CSSs will go through the squeezing stage, much like their isolated cases, apart from some interference terms. Finally, applying a third rotation pulse (optimized in time and angle) around the $x$ axis will split and place one of them to the poles and the other to $\pm y$ axes, generating a $N=4$ state with a cross-legged cat construction of the target template $[(|Z\rangle+|-Z\rangle)-(|Y\rangle+i|-Y\rangle)]$.

Figure 5 illustrates the fidelity with respect to this target state for the $\eta=1$ case of $I=5 / 2$. Here, almost full-swing oscillations are observed at an angular frequency of $\omega_{2}=$ $2 \pi\left(4 \sqrt{7} f_{Q} / 3\right)$. The time-evolving $N=4$ state can indeed be approximately represented by a rotating equator-bound cat state (dashed lines in Fig. 5) with respect to a polar-bound one in the form of

$$
\left[(|Z\rangle+|-Z\rangle)+e^{i \omega_{2} t}(|Y\rangle+i|-Y\rangle)\right] .
$$

The Hamiltonian in Eq. (1) for $\eta=1$ which corresponds to two-axis countertwisting has recently been shown to be amenable for a closed-form solution up to $I=21 / 2$ [52]. Hence, our preference for the $I=5 / 2$ system is due to its strictly periodic (as opposed to quasiperiodic) time evolution [31], stemming from two zero eigenfrequencies, and the other two at $\pm \omega_{1}=2 \pi\left(2 \sqrt{7} f_{Q} / 3\right)$ [52]. The intriguing point here is that the fidelity oscillation of the $N=4$ state occurs at its second harmonic, $\omega_{2}=2 \omega_{1}$. In the Appendix we give the details on obtaining the explicit form of the time evolution operator, where it is shown that the doubly degenerate spectrum is responsible for the strong second-harmonic content. This is observed, for instance, in the time evolution of fidelity by the solid red line in Fig. 5, compared to the no-rotation-pulse case that also displays the fundamental frequency $\omega_{1}$. The ratio of second harmonic to fundamental under $\hat{H}_{\eta=1}$ depends on the location of the initial CSS over the Bloch sphere, which is depicted in Fig. 6. In fact, this ratio becomes unity (actually meaning a frequency doubling) for CSS launched from $| \pm Y\rangle$ or $| \pm Z\rangle$ both coinciding with the countertwisting axes of $\hat{H}_{1}$. Therefore, we have $\omega_{2}=2 \omega_{1}$ as the rotation frequency within the $N=4$ components of the target state. From a practical point of view, this internal rotation offers an additional phase that can be benefited as an extra degree of freedom [37].

\section{E. Decoherence in cat states}

So far, our treatment was rather ideal other than considering the parameter sensitivity of our cat-state generation protocol. Now we would like to address the question of
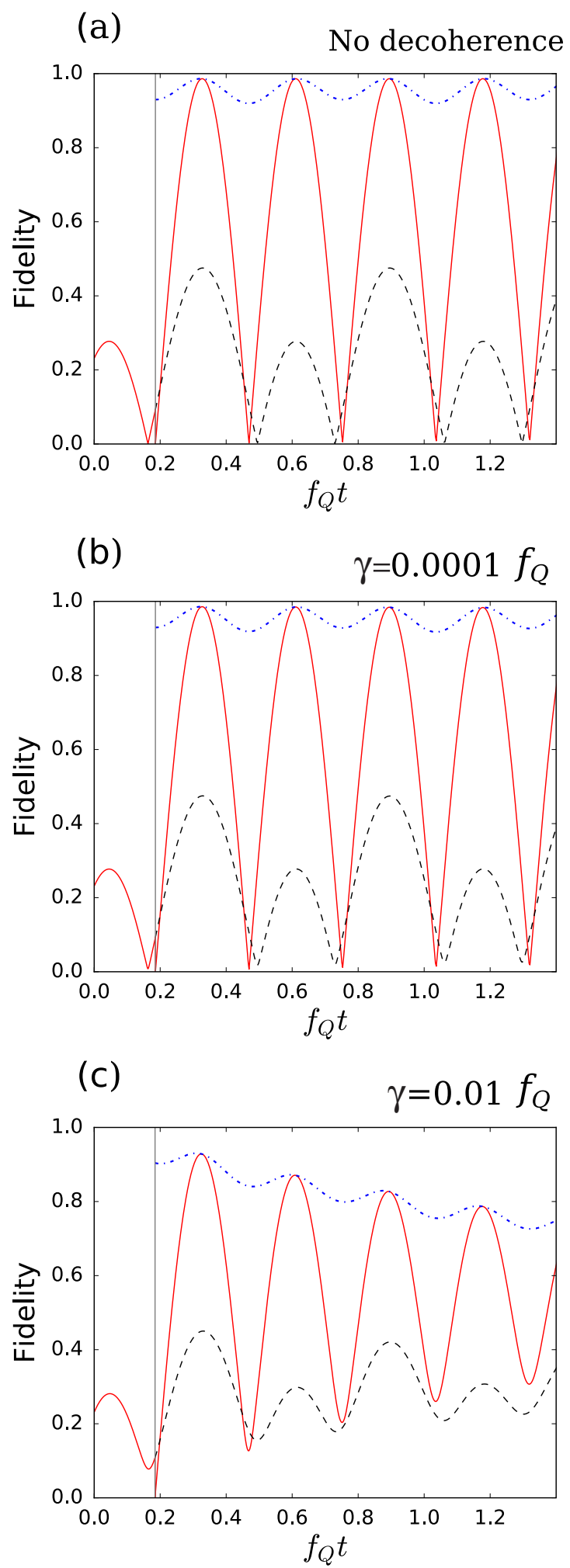

FIG. 5. Fidelity evolution without (black dashed line) and with (red solid line) rotation pulse, the latter resulting in a $N=4$ cat state for $\eta=1$ case of $I=5 / 2$. Also shown is the fidelity with respect to a one-leg rotating target (blue dash-dot line) described by Eq. (14). The vertical gray line marks the instant of the rotation pulse. (a) Without any decoherence included, (b) and (c) with phase damping rates of $\gamma=10^{-4} f_{Q}$ and $10^{-2} f_{Q}$, respectively. 

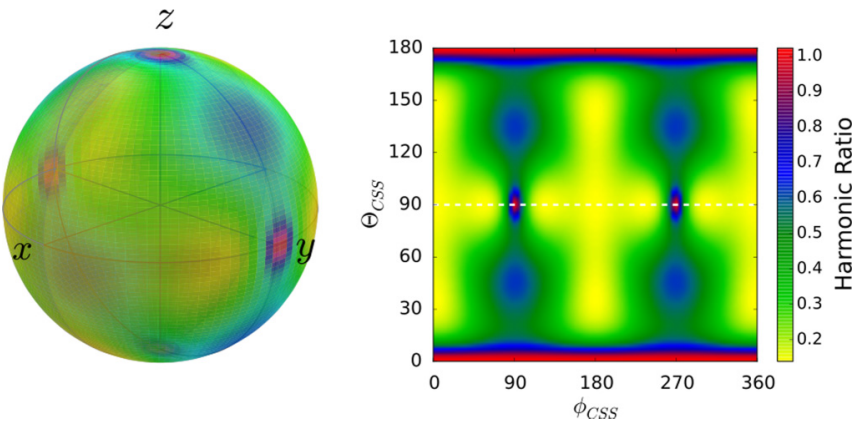

FIG. 6. The ratio of the second-harmonic content with respect to fundamental in the evolution of fidelity as a function of the initial CSS location over the Bloch sphere for $I=5 / 2$.

how good this recipe is in terms of practical realizability, predominantly when it is treated as an open system subject to environmental decoherence. As a matter of fact, from an experimental perspective it is well known that maintaining quantum coherence becomes exceedingly challenging as the distance between the superposed components of the cat state is increased [53]. One advantage of nuclear-spin systems is their immunity to dissipative channels of spontaneous emission at radio frequencies [41] and the particle loss in contrast to cat states produced by Bose-Einstein condensates [27,54], or cavity or circuit quantum electrodynamics $[55,56]$.

In the presence of decoherence, the objective is to assure that the coupling of the nuclear spin to the intended degree of freedom is stronger than that of the dominant environmental process [57]. In our context these are the quadrupolar fre- quency $f_{Q}$ versus the damping rate $\gamma$. The prevalent channel for the latter, in neutral solid-state spin systems (i.e., free from hyperfine coupling to the confined electronic spin) is the phase damping [58]. For quantum dot structures of quadrupolar nuclei (e.g., ${ }^{69,71} \mathrm{Ga},{ }^{75} \mathrm{As},{ }^{115} \mathrm{In}$ ) the dephasing times $\left(T_{2}=1 / \gamma\right)$ lie in the $1-5-\mathrm{ms}$ range [58-60]. For the same systems the quadrupolar frequency dictated by strain is typically in the range $f_{Q} \approx 2-8 \mathrm{MHz}[38,61,62]$. In the case of $\mathrm{NV}$ defect centers, the quadrupolar ${ }^{14} \mathrm{~N}$ nuclear-spin dephasing times are at least $1 \mathrm{~ms}[63]$ and the extracted $f_{Q}$ value is about $10 \mathrm{MHz}[63,64]$. Thus, these two markedly distinct systems share highly similar values for $f_{Q} / \gamma=f_{Q} T_{2} \approx 10^{3}-10^{4}$, suggesting that strong quadrupolar coupling is attainable for such nuclear spins. We should note that there exist solid-state systems with even superior immunity to decoherence, such as the single-crystal $\mathrm{KClO}_{3}$ that has $f_{Q}=28.1 \mathrm{MHz}$ and $T_{2}=4.6 \mathrm{~ms}$ with the product $f_{Q} T_{2}>10^{5}$ [65]. As a caveat, if a nearby unpaired electron spin is present during the stabilization stage, it can degrade nuclear-spin coherence [11].

In the following, we consider dephasing rates $\gamma / f_{Q}$ ranging from $10^{-4}$ to $10^{-2}$, which allows for harsher decoherence to observe its adverse consequences. In Fig. 7 we display how fidelity and rQFI of polar-bound $N=2$ cat states are affected from decoherence for $I=5 / 2$ and $\eta=1$. The Wigner distributions on the right panel refer to $t=10 t_{R}$, i.e., at 10 times the rotation pulse instant, $t_{R}$. As would be anticipated from the strong quadrupolar regime, the case for $\gamma=10^{-4} f_{Q}$ is virtually indistinguishable within this time frame from the decoherence-free ones in Figs. 2(d) and 2(g). For $\gamma=10^{-3} f_{Q}$ the deviation becomes noticeable, and this gets drastic for $\gamma=$ $10^{-2} f_{Q}$, especially on the fidelity, whereas the normalized

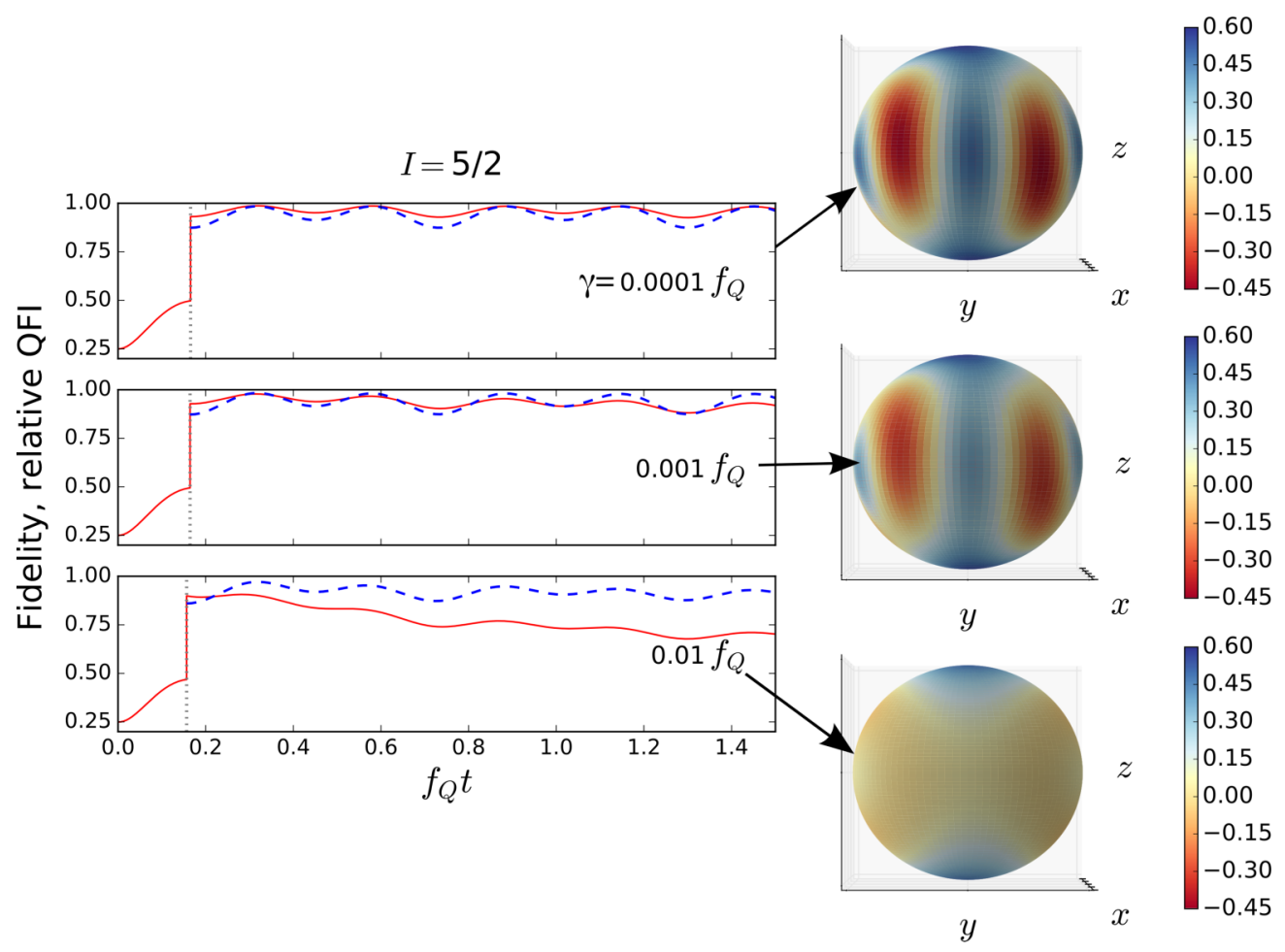

FIG. 7. For $I=5 / 2, \eta=1$, and polar-bound $N=2$ cat state the effect of decoherence. Left panel: Fidelity (solid red line) and normalized rQFI given by Eq. (6) (blue dash line). Right panel: Spin Wigner quasiprobability distributions at $10 t_{R}$. Three different dephasing rates are considered, $\gamma / f_{Q}=10^{-4}, 10^{-3}$, and $10^{-2}$ from top to bottom, respectively. 
rQFI measure [see Eq. (6)] is much less affected as it is insensitive to phase coherence and rather probes the separation of the CSSs. The Wigner distributions are also instrumental in tracking these differences through the attained negative values [66], which are in general taken as a measure of the quantumness of the states [49]. It is clearly seen that decoherence gradually removes these negative interference fringes of the superposition. We note that the equator-bound $N=2$ cat states (not shown) are somewhat less susceptible than the polar-bound ones.

In the same vein, we return to Fig. 5 to discuss how decoherence affects $N=4$ cat states, which reassures us that a rate of $\gamma=10^{-4} f_{Q}$ is not influential whereas $10^{-2} f_{Q}$ becomes very destructive on the fidelity by washing away the contrast between originally orthogonal states. Here, the point to note is that $N=4$ cat states do not particularly suffer more from decoherence than $N=2$ variants. Based on these insights we can conclude that the proposed scheme is decoherence tolerant for rates around $\gamma / f_{Q} \approx 10^{-4}$, that is, in the upper end of currently available range without any additional countermeasures such as the dynamical decoupling of the environmental spins [67].

According to the qudit dephasing model employed in this work, the number of channels increases in proportion to spin angular momentum $I$, as can be seen from Eqs. (7) and (8). Furthermore, we are dealing with quite unique macroscopic quantum spin states that are not necessarily governed by the same dephasing rate $\gamma$ which applies to Dicke states [55]. Therefore, we need to identify how the baseline dephasing rate $\gamma$ compares with the decay rate of cat-state fidelities as a function of $I$. The inset in Fig. 8 displays a typical damping of fidelity under decoherence (here, for $\gamma=10^{-2} f_{Q}$ ), which has an oscillatory pattern for the specific $\eta=0.5$ and $I=5 / 2$ values considered. Its time constant $\tau$ can be extracted by fitting the fidelity to a form $F(t)=F_{0} \exp (-t / \tau)+F_{\text {sat }}$. Figure 8 illustrates the scaling of the fidelity decay time constant as a function of $I$ for the $N=2$ cat states. It reveals that at the lower end of $I$ the fidelity decay rate $(1 / \tau)$ approaches toward $\gamma$. As $I$ increases, its scaling lies roughly in between $I^{3}$ and $I^{5 / 2}$, for $\eta=0.1$ and $\eta=1$, respectively. The

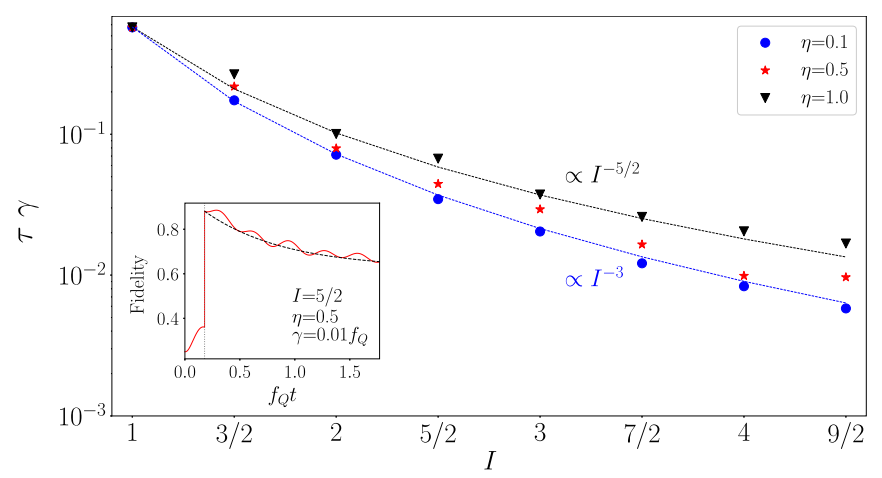

FIG. 8. The variation of fidelity decay time constant $\tau$ (in units of reciprocal dephasing rate, $1 / \gamma)$ with spin $I$. Polar-bound $N=2$ cat states are considered. Dashed lines mark the $I^{-5 / 2}$ and $I^{-3}$ scalings. Inset shows the damping of fidelity after the rotation pulse for the case of $I=5 / 2, \eta=0.5$, and $\gamma=10^{-2} f_{Q}$; dashed line is the exponential fit to extract the decay time constant. overall behavior is essentially preserved for different baseline dephasing rates in the range $\gamma=10^{-2}-10^{-4}$. The enhanced susceptibility to decoherence of the large- $I$ spin cat states is already known, like in the context of spinor Bose-Einstein condensates [68]. A trivial advantage of nuclear spins is that the $I$ values are inherently capped before this scaling becomes prohibitive. Therefore, we believe that there exits opportunities for spin cat states within this range of low angular momenta.

\section{F. Discussions on practical aspects}

Finally, we would like to address key practical issues in the realization of this theoretical proposal in regard to initialization, manipulation, and readout. As the exemplary platform we have particularly in mind the NV centers [57]. We begin with the initialization of the nuclear-spin state. Our aforementioned cat-state generation schemes start from the $|+X\rangle$ CSS, which is accessible from, say, the $|I, I\rangle$ Dicke state by a $\pi / 2$ rotation. In negatively charged defect centers, a nuclear-spin state like $|I, I\rangle$ can be initialized with high fidelity by transferring the polarization from the ancillary electron spin [69]. The latter is easily obtained by means of optically induced electronic spin orientation [70]. Most commonly the transfer of polarization from the electronic to nuclear spin is mediated by the hyperfine interaction [71]. But, alternatively using a forbidden transition with a two-photon process was also proposed [63].

After initialization and a brief interval of QI evolution, the rotation around one of the principal EFG axes is the next manipulation to be imposed on the nuclear spin. Traditionally rotation is accomplished by Rabi flopping through a resonant rf pulse of amplitude and duration to satisfy the pulse area for the desired rotation angle [41]. The nuclear magnetic resonance community has developed measures to attain the intended rotation in the presence of systematic frequency or pulse duration errors [35]. A different choice is to rotate the nuclear spin via off-resonant Rabi oscillation of the electron spin and the hyperfine interaction [72]. This has been experimentally tested on NV centers to yield about 2 orders of magnitude shorter rotation duration compared to the traditional approach with moderate pulse amplitudes [73]. As a matter of fact, fast spin rotation is highly desirable for our framework where we assume it to be instantaneous. Indeed, a few hundred nanosecond nuclear-spin rotation time scale [73] would meet this requirement against microsecond-long durations of QI.

The final stage is the detection, where a projective measurement is performed conventionally on the observable $I_{z}$. However, with prior rotations $I_{x}$ or $I_{y}$ can likewise be read out. As a matter of fact, using a sequence of rotation pulses, measuring the free-induction decay signals, and Fourier transforming them forms the recipe to extract all entries of the density matrix with real and imaginary parts [74]. This is known as quantum state tomography, which has been in use for the nuclear spins for the past two decades. As a relevant example the density matrix reconstruction of three entangled nuclear spins with a nondemolition readout in NV centers can be given $[69,75]$. Moreover, state tomography has been extended to the quadrupolar nuclei [76] and there are also efforts to implement it with small or zero static magnetic field, which in effect makes it the nuclear quadrupole resonance [65]. 
In general, once the full density matrix is at one's disposal by state tomography, the Wigner distributions, as in this work, can be directly computed for target state comparison; a recent such demonstration combining experiment is given in Ref. [30]. Depending on the encoding strategy, rather than the projective $I_{z}$ measurement, a different detection, like the parity of the superposed cat states, is another option [77].

It needs to be mentioned that the involvement of neighboring spins, including the ancillary electron spin, albeit with all its aforestated advantages, gives rise to measurement errors [11-13,15]. Specifically in the case of electron spin, the component of the hyperfine tensor perpendicular to the NV axis causes stochastic quantum jumps in the nuclear spin. Therefore, one should assure the mean jump interval to be longer than the measurement time [11].

An important matter that remains is the role of entanglement. The Schrödinger cat states have long been advocated as the key enabler for quantum sensing to surpass the standard quantum limit [27]. The common framework, as in two-component Bose-Einstein condensates, hinges upon $N$-particle entanglement in constructing a spin- $N / 2$ collective Hilbert space [54]. On the other hand, for the paradigm considered in this work, the entanglement of nuclei plays no role, as it is based on the pure state of a single nuclear spin [78]. The fact that it is a quadrupolar nucleus avails a qudit structure, also granting its controllability via the quadratic Hamiltonian of Eq. (1) that facilitates squeezing [31] and cat-state generation as in this work. Achieving all of these without a need for entanglement with other nuclei makes it less fragile under decoherence, as elucidated in the above analysis. Such an ability to generate and control cat states on a nuclear spin with $I \geqslant 1$ amounts to a small-scale quantum information processor, much like other prototypes that can be put to use in various ways [79]. For expanding our approach, the route of exploiting yet higher level of superpositions (i.e., $N>4$ ) of the cat states while remaining in the same spin angular momentum subspace is blocked because of the limited number of fixed points over the Bloch sphere of the Hamiltonian (in our case, QI). Hence, if the scalability is the primary objective, one should bring in an additional layer of spin-spin interaction among nuclei to harness entanglement through an enlarged Hilbert space [80].

\section{CONCLUSIONS}

In summary, we present a blueprint for generating stabilized nuclear-spin cat states using biaxial QI together with one or three rotation pulses. A rudimentary optimization of the single-pulse approach attains fidelities around 0.95, while being largely insensitive to the variations in the parameters. After analyzing the polar- and equator-bound $N=2$ cat states separately, we considered their superposition with four CSSs, where one of the two-component cat states rotates with respect to the EFG axes. Such states play a crucial role in cat codes to protect against bit flips [81]. To render our analysis more realistic, the effect of phase noise, which is the dominant decoherence mechanism, is thoroughly investigated, showing that these generated cat states can retain their fidelities on the favorable end of the currently accessible decoherence levels.

We believe that optically addressable color centers involving a quadrupolar nucleus, like an implanted indium defect within a wide-band-gap host [82], appears to be a suitable physical system for realizing nuclear-spin cat states. The other option of self-assembled quantum dots possesses an ensemble of more than ten thousands of quadrupolar nuclei that can be to some extent entangled though the confined electron spin [83,84]. The primary challenge here is the spatially inhomogeneous strain causing the tilting variations of EFG axes within a solid-state matrix $[38,85]$. Therefore, a means for narrowing this distribution may be valuable to gain better control over this resource. As other possible extensions, schemes for further increasing the maximum fidelity together with low ripple can be sought to meet the stringent practical demands [86]. This is particularly relevant for the recently introduced cat codes utilizing microwave photons in superconducting circuits, which have proven to be practical for quantum error correction [81]. Therefore, their nuclear-spin cat-state implementation may be pursued both theoretically and experimentally.

\section{ACKNOWLEDGMENTS}

We are grateful to T. Opatrný for fruitful discussions. This work was supported by TÜBITAK, The Scientific and Technological Research Council of Turkey through Project No. 114F409. The numerical calculations reported in this paper were partially performed at TÜBITAK ULAKBIM, High Performance and Grid Computing Center (TRUBA resources).

\section{APPENDIX: TIME EVOLUTION OPERATOR FOR SPIN 5/2}

In this section our aim is to obtain the closed-form expression for the time evolution operator under the QI Hamiltonian of a spin-5/2 system, specifically, for $\eta=1$. The Hamiltonian in Eq. (1) reduces in this case to

$$
\hat{H}_{\eta=1}=\frac{\hbar \omega_{Q}}{3}\left(\hat{I}_{z}^{2}-\hat{I}_{y}^{2}\right)
$$

where $\omega_{Q}=2 \pi f_{Q}$, and the associated characteristic polynomial for $H_{1}$ is given by [52]

$$
p_{H_{1}}(\lambda)=\lambda^{2}\left(\lambda^{2}-28\right)^{2} .
$$

Thus, the six roots are composed of one at zero, and two equal in magnitude but opposite sign eigenfrequencies, each of them being doubly degenerate. That is, the distinct spectrum is composed of $\lambda_{j}=\left\{0, \omega_{1},-\omega_{1}\right\}$, with $\omega_{1}=2 \sqrt{7} \omega_{Q} / 3$. Taking into account the degeneracies in the spectrum [87], we can work out the time evolution operator under $\hat{H}_{1}$ explicitly, starting from

$$
e^{-i \hat{H}_{1} t / \hbar}=\sum_{j=1}^{3} e^{-i \lambda_{j} t} \prod_{\substack{k=1 \\ k \neq j}}^{3} \frac{i \hat{H}_{1} t-\lambda_{k} \hat{1}}{\lambda_{j}-\lambda_{k}},
$$

where $\hat{1}$ is the identity operator. After inserting the eigenfrequencies it leads to the following closed-form expression:

$$
e^{-i \hat{H}_{1} t / \hbar}=\frac{\cos \left(\omega_{1} t\right)-1}{\omega_{1}^{2}} \hat{H}_{1}^{2}-\frac{i \sin \left(\omega_{1} t\right)}{\omega_{1}} \hat{H}_{1}+\hat{1} .
$$

As mentioned in the main text, the double degeneracy in the spectrum gives rise to second-harmonic generation with respect to the fundamental eigenfrequency of $\omega_{1}$. 
[1] J. P. Dowling and G. J. Milburn, Quantum technology: The second quantum revolution, Philos. Trans. R. Soc., A 361, 1655 (2003).

[2] D. G. Cory, A. F. Fahmy, and T. F. Havel, Ensemble quantum computing by NMR spectroscopy, Proc. Natl. Acad. Sci. USA 94, 1634 (1997).

[3] B. E. Kane, A silicon-based nuclear spin quantum computer, Nature (London) 393, 133 (1998).

[4] M. H. Levitt, Spin dynamics: Basics of Nuclear Magnetic Resonance (John Wiley \& Sons, New York, 2007).

[5] W. S. Warren, The usefulness of NMR quantum computing, Science 277, 1688 (1997).

[6] J. Köhler, J. A. J. M. Disselhorst, M. C. J. M. Donckers, E. J. J. Groenen, J. Schmidt, and W. E. Moerner, Magnetic resonance of a single molecular spin, Nature (London) 363, 242 (1993).

[7] J. Wrachtrup, C. Von Borczyskowski, J. Bernard, M. Orritt, and R. Brown, Optical detection of magnetic resonance in a single molecule, Nature (London) 363, 244 (1993).

[8] J. Wrachtrup, A. Gruber, L. Fleury, and C. Von Borczyskowski, Magnetic resonance on single nuclei, Chem. Phys. Lett. 267, 179 (1997).

[9] F. Jelezko, T. Gaebel, I. Popa, M. Domhan, A. Gruber, and J. Wrachtrup, Observation of Coherent Oscillation of a Single Nuclear Spin and Realization of a Two-Qubit Conditional Quantum Gate, Phys. Rev. Lett. 93, 130501 (2004).

[10] M. V. G. Dutt, L. Childress, L. Jiang, E. Togan, J. Maze, F. Jelezko, A. S. Zibrov, P. R. Hemmer, and M. D. Lukin, Quantum register based on individual electronic and nuclear spin qubits in diamond, Science 316, 1312 (2007).

[11] P. Neumann, J. Beck, M. Steiner, F. Rempp, H. Fedder, P. R. Hemmer, J. Wrachtrup, and F. Jelezko, Single-shot readout of a single nuclear spin, Science 329, 542 (2010).

[12] L. Robledo, L. Childress, H. Bernien, B. Hensen, P. F. A. Alkemade, and R. Hanson, High-fidelity projective read-out of a solid-state spin quantum register, Nature (London) 477, 574 (2011).

[13] A. Dréau, P. Spinicelli, J. R. Maze, J.-F. Roch, and V. Jacques, Single-Shot Readout of Multiple Nuclear Spin Qubits in Diamond Under Ambient Conditions, Phys. Rev. Lett. 110, 060502 (2013).

[14] R. Vincent, S. Klyatskaya, M. Ruben, W. Wernsdorfer, and F. Balestro, Electronic read-out of a single nuclear spin using a molecular spin transistor, Nature (London) 488, 357 (2012).

[15] J. J. Pla, K. Y. Tan, J. P. Dehollain, W. H. Lim, J. J. L. Morton, F. A. Zwanenburg, D. N. Jamieson, A. S. Dzurak, and A. Morello, High-fidelity readout and control of a nuclear spin qubit in silicon, Nature (London) 496, 334 (2013).

[16] D. Suter and F. Jelezko, Single-spin magnetic resonance in the nitrogen-vacancy center of diamond, Prog. Nucl. Magn. Reson. Spectrosc. 98-99, 50 (2017).

[17] J. M. Radcliffe, Some properties of coherent spin states, J. Phys. A 4, 313 (1971).

[18] M. Kitagawa and M. Ueda, Squeezed spin states, Phys. Rev. A 47, 5138 (1993).

[19] J. Ma, X. Wang, C. P. Sun, and F. Nori, Quantum spin squeezing, Phys. Rep. 509, 89 (2011).

[20] V. V. Dodonov, I. A. Malkin, and V. I. Man'Ko, Even and odd coherent states and excitations of a singular oscillator, Physica 72, 597 (1974)
[21] B. Yurke and D. Stoler, Generating Quantum Mechanical Superpositions of Macroscopically Distinguishable States Via Amplitude Dispersion, Phys. Rev. Lett. 57, 13 (1986).

[22] T. Opatrný and K. Mølmer, Spin squeezing and Schrödingercat-state generation in atomic samples with Rydberg blockade, Phys. Rev. A 86, 023845 (2012).

[23] H. W. Lau, Z. Dutton, T. Wang, and C. Simon, Proposal for the Creation and Optical Detection of Spin Cat States in BoseEinstein Condensates, Phys. Rev. Lett. 113, 090401 (2014).

[24] S. Dooley, F. McCrossan, D. Harland, M. J. Everitt, and T. P. Spiller, Collapse and revival and cat states with an $n$-spin system, Phys. Rev. A 87, 052323 (2013).

[25] S. Dooley and T. P. Spiller, Fractional revivals, multipleSchrödinger-cat states, and quantum carpets in the interaction of a qubit with $n$ qubits, Phys. Rev. A 90, 012320 (2014).

[26] J. Huang, X. Qin, H. Zhong, Y. Ke, and C. Lee, Quantum metrology with spin cat states under dissipation, Sci. Rep. 5, 17894 (2015).

[27] L. Pezzè, A. Smerzi, M. K. Oberthaler, R. Schmied, and P. Treutlein, Non-classical states of atomic ensembles: fundamentals and applications in quantum metrology, arXiv:1609.01609.

[28] M. H. Cohen and F. Reif, Quadrupole effects in nuclear magnetic resonance studies of solids, Solid State Phys. 5, 321 (1957).

[29] T. P. Das and E. L. Hahn, Nuclear Quadrupole Resonance Spectroscopy (Academic Press, New York, 1958).

[30] R. Auccaise, A. G. Araujo-Ferreira, R. S. Sarthour, I. S. Oliveira, T. J. Bonagamba, and I. Roditi, Spin Squeezing in a Quadrupolar Nuclei NMR System, Phys. Rev. Lett. 114, 043604 (2015).

[31] Y. Aksu Korkmaz and C. Bulutay, Nuclear spin squeezing via electric quadrupole interaction, Phys. Rev. A 93, 013812 (2016).

[32] G.-R. Jin and S. W. Kim, Storage of Spin Squeezing in a Two-Component Bose-Einstein Condensate, Phys. Rev. Lett. 99, 170405 (2007).

[33] L.-N. Wu, M. K. Tey, and L. You, Persistent atomic spin squeezing at the Heisenberg limit, Phys. Rev. A 92, 063610 (2015).

[34] D. Kajtoch, K. Pawłowski, and E. Witkowska, Entanglement storage by classical fixed points in the two-axis countertwisting model, Phys. Rev. A 93, 022331 (2016).

[35] H. K. Cummins, G. Llewellyn, and J. A. Jones, Tackling systematic errors in quantum logic gates with composite rotations, Phys. Rev. A 67, 042308 (2003).

[36] M. Mirrahimi, Z. Leghtas, V. V. Albert, S. Touzard, R. J. Schoelkopf, L. Jiang, and M. H. Devoret, Dynamically protected cat-qubits: A new paradigm for universal quantum computation, New J. Phys. 16, 045014 (2014).

[37] V. V. Albert, C. Shu, S. Krastanov, C. Shen, R.-B. Liu, Z.-B. Yang, R. J. Schoelkopf, M. Mirrahimi, M. H. Devoret, and L. Jiang, Holonomic Quantum Control with Continuous Variable Systems, Phys. Rev. Lett. 116, 140502 (2016).

[38] C. Bulutay, Quadrupolar spectra of nuclear spins in strained $\mathrm{in}_{x} \mathrm{ga}_{1-x}$ as quantum dots, Phys. Rev. B 85, 115313 (2012).

[39] B. C. Sanders, Quantum dynamics of the nonlinear rotator and the effects of continual spin measurement, Phys. Rev. A 40, 2417 (1989).

[40] A. Gilchrist, K. Nemoto, W. J. Munro, T. C. Ralph, S. Glancy, S. L. Braunstein, and G. J. Milburn, Schrödinger cats and their power for quantum information processing, J. Opt. B: Quantum Semiclassical Opt. 6, S828 (2004). 
[41] C. C. Gerry and P. L. Knight, Introductory Quantum Optics (Cambridge University Press, Cambridge, UK, 2005).

[42] M. A. Nielsen and I. L. Chuang, Quantum Computation and Quantum Information (Cambridge University Press, New York, 2000).

[43] G. Björk and P. G. Luca Mana, A size criterion for macroscopic superposition states, J. Opt. B: Quantum Semiclassical Opt. 6, 429 (2004).

[44] F. Fröwis and W. Dür, Measures of macroscopicity for quantum spin systems, New J. Phys. 14, 093039 (2012).

[45] S. Pirandola, S. Mancini, S. L. Braunstein, and D. Vitali, Minimal qudit code for a qubit in the phase-damping channel, Phys. Rev. A 77, 032309 (2008).

[46] Y.-X. Huang, J. Ma, X.-X. Jing, and X.-G. Wang, Spin squeezing and fixed-point bifurcation, Commun. Theor. Phys. 58, 800 (2012).

[47] J. R. Johansson, P. D. Nation, and Franco Nori, Qutip: An opensource python framework for the dynamics of open quantum systems, Comput. Phys. Commun. 183, 1760 (2012).

[48] J. R. Johansson, P. D. Nation, and Franco Nori, Qutip2: A Python framework for the dynamics of open quantum systems, Comput. Phys. Commun. 184, 1234 (2013).

[49] G. S. Agarwal, R. R. Puri, and R. P. Singh, Atomic Schrödinger cat states, Phys. Rev. A 56, 2249 (1997).

[50] S. M. Chumakov, A. Frank, and K. B. Wolf, Finite Kerr medium: Macroscopic quantum superposition states and Wigner functions on the sphere, Phys. Rev. A 60, 1817 (1999).

[51] A. Roy, A. D. Stone, and L. Jiang, Concurrent remote entanglement with quantum error correction against photon losses, Phys. Rev. A 94, 032333 (2016).

[52] M. Bhattacharya, Analytical solvability of the two-axis countertwisting spin squeezing Hamiltonian, arXiv:1509.08530.

[53] M. Brune, E. Hagley, J. Dreyer, X. Maître, A. Maali, C. Wunderlich, J. M. Raimond, and S. Haroche, Observing the Progressive Decoherence of the "Meter" in a Quantum Measurement, Phys. Rev. Lett. 77, 4887 (1996).

[54] T. Byrnes, K. Wen, and Y. Yamamoto, Macroscopic quantum computation using Bose-Einstein condensates, Phys. Rev. A 85, 040306 (2012).

[55] S. Haroche and J.-M. Raimond, Exploring the Quantum (Oxford University Press, Oxford, UK, 2006).

[56] A. Wallraff, D. I. Schuster, A. Blais, L. Frunzio, R.-S. Huang, J. Majer, S. Kumar, S. M. Girvin, and R. J. Schoelkopf, Strong coupling of a single photon to a superconducting qubit using circuit quantum electrodynamics, Nature (London) 431, 162 (2004).

[57] C. Müller, X. Kong, J.-M. Cai, K. Melentijević, A. Stacey, M. Markham, D. Twitchen, J. Isoya, S. Pezzagna, J. Meijer, J. F. Du, M. B. Plenio, B. Naydenov, L. P. McGuinness, and F. Jelezko, Nuclear magnetic resonance spectroscopy with single spin sensitivity, Nat. Commun. 5, 4703 (2014).

[58] G. Wüst, M. Munsch, F. Maier, A. V. Kuhlmann, A. Ludwig, A. D. Wieck, D. Loss, M. Poggio, and R. J. Warburton, Role of the electron spin in determining the coherence of the nuclear spins in a quantum dot, Nat. Nanotechnol. 11, 885 (2016).

[59] E. A. Chekhovich, M. Hopkinson, M. S. Skolnick, and A. I. Tartakovskii, Suppression of nuclear spin bath fluctuations in self-assembled quantum dots induced by inhomogeneous strain, Nat. Commun. 6, 6348 (2015).
[60] A. M. Waeber, M. Hopkinson, I. Farrer, D. A. Ritchie, J. Nilsson, R. Ma. Stevenson, A. J. Bennett, A. J. Shields, G. Burkard, A. I. Tartakovskii, M. S. Skolnick, and E. A. Chekhovich, Fewsecond-long correlation times in a quantum dot nuclear spin bath probed by frequency-comb nuclear magnetic resonance spectroscopy, Nat. Phys. 12, 688 (2016).

[61] M. S. Kuznetsova, K. Flisinski, I. Ya. Gerlovin, M. Yu. Petrov, I. V. Ignatiev, S. Yu. Verbin, D. R. Yakovlev, D. Reuter, A. D. Wieck, and M. Bayer, Nuclear magnetic resonances in (In,Ga)As/GaAs quantum dots studied by resonant optical pumping, Phys. Rev. B 89, 125304 (2014).

[62] M. Munsch, G. Wüst, A. V. Kuhlmann, F. Xue, A. Ludwig, D. Reuter, A. D. Wieck, M. Poggio, and R. J. Warburton, Manipulation of the nuclear spin ensemble in a quantum dot with chirped magnetic resonance pulses, Nat. Nanotechnol. 9, 671 (2014).

[63] A. Ajoy and P. Cappellaro, Stable three-axis nuclearspin gyroscope in diamond, Phys. Rev. A 86, 062104 (2012)

[64] X.-F. He, N. B. Manson, and P. T. H. Fisk, Paramagnetic resonance of photoexcited $n-V$ defects in diamond, II. Hyperfine interaction with the ${ }^{14} \mathrm{~N}$ nucleus, Phys. Rev. B 47, 8816 (1993).

[65] J. Teles, C. Rivera-Ascona, R. S. Polli, R. Oliveira-Silva, E. L. G. Vidoto, J. P. Andreeta, and T. J. Bonagamba, Experimental implementation of quantum information processing by Zeemanperturbed nuclear quadrupole resonance, Quantum Inf. Process. 14, 1889 (2015).

[66] C. Joana, P. van Loock, H. Deng, and T. Byrnes, Steady-state generation of negative-Wigner-function light using feedback, Phys. Rev. A 94, 063802 (2016).

[67] D. Suter and G. A. Álvarez, Colloquium: Protecting quantum information against environmental noise, Rev. Mod. Phys. 88, 041001 (2016).

[68] T. Byrnes, D. Rosseau, M. Khosla, A. Pyrkov, A. Thomasen, T. Mukai, S. Koyama, A. Abdelrahman, and E. Ilo-Okeke, Macroscopic quantum information processing using spin coherent states, Opt. Commun. 337, 102 (2015).

[69] G. Waldherr et al., Quantum error correction in a solidstate hybrid spin register, Nature (London) 506, 204 (2014).

[70] N. B. Manson, J. P. Harrison, and M. J. Sellars, Nitrogenvacancy center in diamond: Model of the electronic structure and associated dynamics, Phys. Rev. B 74, 104303 (2006)

[71] B. Smeltzer, J. McIntyre, and L. Childress, Robust control of individual nuclear spins in diamond, Phys. Rev. A 80, 050302 (2009).

[72] J. J. L. Morton, A. M. Tyryshkin, A. Ardavan, S. C. Benjamin, K. Porfyrakis, S. A. Lyon, and G. A. D. Briggs, Bang-bang control of fullerene qubits using ultrafast phase gates, Nat. Phys. 2, 40 (2006).

[73] S. Sangtawesin, T. O. Brundage, and J. R. Petta, Fast RoomTemperature Phase Gate on a Single Nuclear Spin in Diamond, Phys. Rev. Lett. 113, 020506 (2014).

[74] I. L. Chuang, N. Gershenfeld, M. G. Kubinec, and D. W. Leung, Bulk quantum computation with nuclear magnetic resonance: Theory and experiment, Proc. R. Soc. London, Ser. A 454, 447 (1998). 
[75] J. Cramer, N. Kalb, M. A. Rol, B. Hensen, M. S. Blok, M. Markham, D. J. Twitchen, R. Hanson, and T. H. Taminiau, Repeated quantum error correction on a continuously encoded qubit by real-time feedback, Nat. Commun. 7, 11526 (2016).

[76] J. Teles, E. R. deAzevedo, R. Auccaise, R. S. Sarthour, I. S. Oliveira, and T. J. Bonagamba, Quantum state tomography for quadrupolar nuclei using global rotations of the spin system, J. Chem. Phys. 126, 154506 (2007).

[77] R. P. Rundle, P. W. Mills, T. Tilma, J. H. Samson, and M. J. Everitt, Quantum phase space measurement and entanglement validation made easy, arXiv:1605.08922.

[78] Z. Gedik, I. A. Silva, B. Çakmak, G. Karpat, E. L. G. Vidoto, D. O. Soares-Pinto, E. R. deAzevedo, and F. F. Fanchini, Computational speed-up with a single qudit, Sci. Rep. 5, 14671 (2015).

[79] M. Steffen, J. M. Gambetta, and J. M. Chow, Progress, status, and prospects of superconducting qubits for quantum computing, in 2016 46th European Solid-State Device Research Conference (ESSDERC) (IEEE, 2016), pp. 17-20.

[80] M. N. Leuenberger and D. Loss, Grover algorithm for large nuclear spins in semiconductors, Phys. Rev. B 68, 165317 (2003).
[81] N. Ofek et al., Extending the lifetime of a quantum bit with error correction in superconducting circuits, Nature (London) 536, 441 (2016).

[82] B. J. M. Hausmann, T. M. Babinec, J. T. Choy, J. S. Hodges, S. Hong, I. Bulu, A. Yacoby, M. D. Lukin, and M. Lončar, Single-color centers implanted in diamond nanostructures, New J. Phys. 13, 045004 (2011).

[83] W. Yang and R.-B. Liu, Quantum many-body theory of qubit decoherence in a finite-size spin bath, II. Ensemble dynamics, Phys. Rev. B 79, 115320 (2009).

[84] D. Stanek, C. Raas, and G. S. Uhrig, From quantum-mechanical to classical dynamics in the central-spin model, Phys. Rev. B 90, 064301 (2014).

[85] C. Bulutay, E. A. Chekhovich, and A. I. Tartakovskii, Nuclear magnetic resonance inverse spectra of InGaAs quantum dots: Atomistic level structural information, Phys. Rev. B 90, 205425 (2014).

[86] E. Knill, Quantum computing with realistically noisy devices, Nature (London) 434, 39 (2005).

[87] F. De Zela, Closed-form expressions for the matrix exponential, Symmetry 6, 329 (2014). 